\title{
Workshop 1 - Dosimetry in Research and Test Reactors and in Accelerators
}

\author{
Co-chairs: \\ EWGRD: Mladen Mitev (INRNE, Bulgaria) \\ ASTM: David Vehar (Sandia National Laboratories, USA)
}

Based on workshop data from ISRD-14, the body of papers presented at this Symposium, and challenges currently facing the application of dosimetry to Research and Test Reactors Accelerators, three general topics were suggested to workshop attendees:

1. Current Research and Test Reactors activities, including review and documentation of older dosimetry data;

2. The possible contribution of accelerator facilities to the development of reactor dosimetry; and

3. Advances in radiation heating measurements using calorimeters, including possible applications and challenges.

Workshop participants were invited to express their opinions on the suggested topics. Those comments are summarized as follows.

\section{Current Activities}

Considerable effort is being expended on the review and documentation of older data. One motivation for this is the need for customers to duplicate the test conditions for work that may have been performed decades ago. Another is the need to properly archive data for the increasing number of facilities that have already been shut down in Europe, the United States and Japan, or are scheduled to be shut down in the near future.

Other reasons exist, but the inability of facilities to meet performance requirements imposed after the Fukushima event was identified as a major contributor.

Beyond the need to perform extensive review and documentation of experiments already conducted in order to preserve this knowledge for future use, it is important that facilities stopping operation in the near future identify the data that still need to be obtained, and perform the necessary experimental work before the shutdown occurs.

Reactors in operation continue to maintain their usual activities with regard to detector calibration and code validation. Although decreasing in number, there still exist reactors with standard fields for calibration and code validation, such as those at SCK-CEN in Belgium and a new critical assembly under construction in Nevada in the United States. The option to seek access to the reactors in China

This is an Open Access article distributed under the terms of the Creative Commons Attribution License 2.0, which permits unrestricted use, distribution, and reproduction in any medium, provided the original work is properly cited. 
was also suggested, although it was concluded that any intercomparison in the near future would need to be between the facilities in Europe and the United States.

\section{Use of Accelerators}

The prevailing opinion was that accelerators remain useful in nuclear data evaluation and time of flight measurements, but not as much for supporting reactor dosimetry applications. Based on the movement of Boron Neutron Capture Therapy from reactor to accelerator epithermal neutron sources, it was pointed out that the possibility of creating suitable neutron spectra exits, and that accelerator facilities are able to create different spectra using appropriate targets and filters. Several facilities with such abilities are in construction - MAX and SPIRAL for example, and they will provide an opportunity for thermal neutron dosimetry. The extensive dosimetry demands of BNCT was also mentioned.

\section{Calorimeters}

Two main fields of application of radiation heating measurements were identified: in electronics, and in fusion technology. For electronics, where there is a need to distinguish between neutron and gamma-ray effects, calorimetry is being used in conjunction with other available techniques such as TLD, PCD, and activation foils. The most current application in the fusion technology is the evaluation of engineering materials, such as determining energy deposition in magnetic coil insulation.

\section{Open Discussion}

Finally, an open discussion centered around the number of reactors available for materials testing. These include reactors at INEL (USA), SCK-CEN (Belgium), NRI/Rez (Czech Republic) and INR/Świerk (Poland). The LVR-15 in Rez provides open access for performing experiments. In addition, some reactors currently provide training for NPP personnel. It was also noted that the new hybrid acceleratorreactor facilities are being developed, such as the MYRRHA project that promises considerable interest from the industry. 